\title{
A Study of the Electromedics Autotransfusion System CRADA PC93-010, Final Report
}

\author{
Mehrdad Shahnam \\ Franklin Shaffer
}

October 1993 to March 1995

U.S. Department of Energy

Pittsburgh Energy Technology Center

626 Cochrans Mills Road

Pittsburgh, PA 15236

and

Electromedics

7337 S. Revere Parkway

Englewood, CO 80112

RECEIVED

FEB 261999

OSTI 


\section{DISCLAIMER}

This report was prepared as an account of work sponsored by an agency of the United States Government. Neither the United States Government nor any agency thereof, nor any of their employees, make any warranty, express or implied, or assumes any legal liability or responsibility for the accuracy, completeness, or usefulness of any information, apparatus, product, or process disclosed, or represents that its use would not infringe privately owned rights. Reference herein to any specific commercial product, process, or service by trade name, trademark, manufacturer, or otherwise does not necessarily constitute or imply its endorsement, recommendation, or favoring by the United States Government or any agency thereof. The views and opinions of authors expressed herein do not necessarily state or reflect those of the United States Government or any agency thereof. 


\section{DISCLAIMER}

Portions of this document may be illegible in electronic image products. Images are produced from the best available original document. 


\title{
Summary Report for a CRADA between the Pittsburgh Energy Technology Center and Electromedics, Inc.
}

\author{
A STUDY OF THE \\ Mehrdad Shahnam \\ Gilbert Commonwealth \\ Franklin Shaffer \\ U.S. Department of Energy
}

ELECTROMEDICS AUTOTRANSFUSION SYSTEM

\begin{abstract}
This report describes the work at PETC to evaluate flow dynamics in the Electromedics autotransfusion system. First, a literature survey was conducted for flow studies in centrifuge systems. Although no flow studies were identified for Latham-type bowls, pertinent literature for general centrifugal separation was found and reviewed. Sample measurements were taken with a Laser Doppler Velocimetry (LDV). The data indicates that LDV is a useful tool in flow analysis. Velocity, turbulence intensity, and bowl vibration are all accurately measured with $\mathrm{LDV}$.

For optical imaging of particle separation it is necessary to use fluorescent doped particles and color separation. This allows each type of particle to be observed in a mixture. A market survey was completed and sources for fluorescent dyed particles of three different emission wavelengths and corresponding optical bandpass filters were identified.
\end{abstract}




\subsection{INTRODUCTION}

A literature review was conducted for studies of flow dynamics in centrifugal separation systems. Although no flow studies were identified for Latham-type bowls, a ponderous literature exists for general centrifuge systems. Pertinent papers are attached. The theoretical description, as well as the experimental techniques, to describe centrifugal separation were reviewed.

The second part of this report gives a brief description of the theory of centrifuge separation. Only the practical aspects of theory are covered. To this end, the dimensionless similarity parameters necessary to achieve realistic experimental conditions are listed. It is also shown that particle separation is governed by particle density during centrifugal separation and particle size during the washing cycle.

The third part of this report pertains to selection of particles for flow studies in the Electromedics centrifuge. A source was found for polystyrene latex particles with sizes corresponding to the different types of blood cells. These particles can also be doped with three different colors of fluorescent dyes excitable with the argon, copper-vapor, and $\mathrm{Nd}$ :Yag lasers available in the Flow Analysis Lab. Sources for optical bandpass filters corresponding to different types of fluorescent dyes were also identified.

The fourth part of this report gives initial results of Laser Doppler Velocimetry (LDV) measurements in the Electromedics. centrifuge. LDV provides an instantaneous point measurement of velocity in two directions. Because of LDV's high data rate statistical information such as turbulence intensities and Reynolds shear stresses can be derived. Also, it was found that a velocity measurement could be made in the wall of the bowl. This allows direct measurement of the statistics of bowl vibration.

Initial tests with $100 \mu \mathrm{m}$ particles also showed that multiple pulse particle tracking was feasible. More investigation is required for particle tracking. Efforts now are focused on LDV.

\subsection{LITERATURE REVIEW}

A literature search was conducted for previous flow studies on latham-type centrifuge bowls. Although no flow studies were found for latham-type bowls, a substantial volume of work was identified dealing with general centrifugal separation of a liquid-particle mixture. A number of these papers were reviewed and copies are attached. The majority of these studies were focused on the theoretical modeling of centrifugal separation in cylindrical and conical containers with or without end plates. Experimental studies were limited to simple photography of the separation process yielding only qualitative information on the shape and motion of various layers of separated materials. 


\subsection{BASIC DESIGN THEORY}

The efficiency of centrifugal separation of a mixture is governed by numerous forces including coriolis, inertia and viscous. Each can influence the centrifugal separation in a different manner. For example, in centrifugal separation of a mixture in cylindrical vessels, an increase in the corilois forces causes an azimuthal retrograde velocity for the heavier particles relative to lighter ones resulting in a lowered separation efficiency. Thus, it is critically important to understand the forces dominating the separation process. By surveying the work done in centrifugal separation, it was found that a series of nondimensional parameters can be derived to characterize flow dynamics in centrifugal separation. Although all of the studies were done in container geometries other than the a latham-type bowl, the governing non-dimensional parameters still apply.

To achieve a dynamic similarity between our laboratory experiment and the real transfusion process certain dimensionless parameters must be properly matched. In practical terms, this means that the physical characteristics of the optically transparent blood analog fluid (rheology, density, etc.) and the physical characteristics of tracer particles (size, shape, density etc.) must be carefully chosen based on the pertinent dimensionless parameters.

The process of centrifugal separation is governed by the following non dimensionless parameters:

Ekman number $=\frac{v}{\omega r_{0}^{2}}$; the influence of viscosity and coriolis force

Taylor number $=\frac{\rho \omega}{D}$; the ratio of coriolis force to drag force

Rossby number $=\frac{U_{0}}{\omega r_{o}}$; the ratio of inertia to coriolis forces

$\varepsilon=\frac{\rho_{D}-\rho_{C}}{\rho_{C}} ;$ the density difference between the dispersed and continuous phases

The equation of motion for a spherical particle in a flow is given as

$\frac{d \vec{v}_{p}}{d t}=\frac{18 \mu}{\mathrm{d}_{\mathrm{p}}^{2} \rho_{\mathrm{p}}}\left(\vec{v}_{g}-\vec{v}_{p}\right)+2 \frac{\rho_{g}}{\rho_{\mathrm{p}}}\left(\frac{d \vec{v}_{g}}{d t}-\frac{d \vec{v}_{p}}{d t}\right)+\frac{9}{\pi \mathrm{d}_{\mathrm{p}} \rho_{\mathrm{p}}} \sqrt{\pi \rho_{g} \mu} \int_{t_{o}}^{t} \frac{\frac{d}{d t_{o}}\left(\vec{v}_{g}-\vec{v}_{p}\right)}{\sqrt{t-t_{o}}} d t_{o}+$ (I)

(II)

(III)

(IV)

$$
\frac{\rho_{g}}{\rho_{\mathrm{p}}} \frac{d \bar{v}_{g}}{d t}-\vec{g}\left(1-\frac{\rho_{g}}{\rho_{p}}\right)+\overrightarrow{\mathrm{F}}_{\mathrm{b}}\left(1-\frac{\rho_{g}}{\rho_{p}}\right)
$$

(VI)

(VII)

where

(I) is the force/unit mass required to accelerate the particle.

(II) is the viscous drag force/unit mass using the Stokes' law. 
(III) is the force/unit mass due to the acceleration of the apparent mass of the particle relative to the fluid.

(IV) is the Basset term/unit mass which accounts for deviation from the steady state flow pattern.

(V) is the force/unit mass due to the pressure gradient in the surrounding fluid caused by the acceleration of the fluid.

(VI) is the gravitational acceleration

(VII) is the external force/unit mass (i.e. centrifugal acceleration).

Since in this study the force due to the centrifugal acceleration of a particle, (VII), is many order of magnitiute larger than other forces (III), (IV), (V), and (VI) the latter forces can be neglected reducing the equation of motion for a spherical particle flow to

$$
\frac{d \vec{v}_{p}}{d t}=\frac{18 \mu}{\mathrm{d}_{\mathrm{p}}^{2} \rho_{\mathrm{p}}}\left(\vec{v}_{g}-\vec{v}_{p}\right)+\overrightarrow{\mathrm{F}}_{\mathrm{b}}\left(1-\frac{\rho_{g}}{\rho_{p}}\right)
$$

where $F_{b}=\frac{v_{\theta}^{2}}{R}$ is the centrifugal acceleration.

It is evident from equation 2.2 that density difference between the dispersed and the continuous phases is an important factor effecting the centrifugal force and subsiquently the radial separation of particles. In the wash cycle of the process, however, viscous drag force (first term on the left hand side of equation 2.2) will have more influence than it does in the separation cycle.

\subsection{TRACER PARTICLE SELECTION}

Two types of tracer particles are needed for two types of measurements. To simulate the motion of blood cells fluorescent particles are needed. These particles must mimic the motion of different types of blood cells. To map out the fluid flow with LDV, particles are needed that both follow the fluid flow and disperse uniformly throughout the fluid.

Because of the special shapes of blood cells, finding particles that mimic the motion of blood cells will be difficult. The simulate blood cell motion tracer particles with an "effective equivalent aerodynamic diameter" [1] must be used. Since we desire to use fluorescent-dyed particles we are limited to spherical particles made of polystyrene latex. More investigation is needed to properly choose tracer particle sizes.

Fluorescent dyed particles are necessary to image the flow close to the walls of the centrifuge. Although the density of these particles is fixed at $1.05 \mathrm{~g} / \mathrm{cc}$, it is possible to have these particles made in specific sizes.

Once suitable particles are chosen, it is possible to dye each size with a different color of fluorescent dye. The separation of the different types of particles could be distinguished by color. An extensive market survey was conducted by a high school teacher on a summer internship program in the Flow Analysis Lab. Three small companies were found that manufacture fluorescent particle but only one company was able to use 
dyes of different emission colors that are excitable with the green laser light used in the Flow Analysis lab. The three dyes and emission and excitation peaks are listed below.

\begin{tabular}{|c|c|c|}
\hline Name & Excitation Range (nm) & Emission Peak (nm) \\
\hline fluo-1 & $420-575$ & 655 \\
\hline Propidiam Iodide & 366,488 & 625 \\
\hline "Princeton Red" & 488 & 585 \\
\hline
\end{tabular}

Further testing is needed to determine if these particles will be bright enough to detect. It may be necessary to have the company make a custom batch of particles with maximum dye concentration.

A set of bandpass filters with sharp rolloff will be used to individually observe and measure each particle color. Bandpass filters from numerous companies were reviewed. Below is a table giving the pertinent specifications for bandpass filters corresponding to the dyes listed in the table above.

\begin{tabular}{|c|c|}
\hline $\begin{array}{c}\text { Mean Pass Wavelength } \\
\text { (nm) }\end{array}$ & FWHM (nm) \\
\hline 656.3 & $10 \pm 2$ \\
\hline 620 & $10 \pm 2$ \\
\hline 589.3 & $10 \pm 2$ \\
\hline
\end{tabular}




\subsection{LASER DOPPLER VELOCIMETRY RESULTS}

In order to demonstrate the feasibility of taking velocity measurements inside the latham bowl, a Laser Doppler Velocimeter (LDV) was used to take tangential and axial (up and down) velocity measurements at a cross section about $32 \mathrm{~mm}$ from the bottom of the bowl. 3000 velocity samples at eight different locations, each $1 \mathrm{~mm}$ apart, were taken at this cross section. The bowl was rotating at 762 RPM and the flow rate through the bowl was set at $1000 \mathrm{ml} / \mathrm{min}$. At $762 \mathrm{RPM}$, the expected tangential velocity at the outer wall is about $3.98 \mathrm{~m} / \mathrm{s}$. A mixture of tap water and glycerol was used as the working fluid. Since this experiment was for demonstration purpose only, no attempt was made to match the index of refraction of the fluid with the bowl material. The results are shown in Figures 1 and 2 . As expected, the tangential velocity decreases moving from the outer wall to the inner cone wall, Figure 1. It can be seen from Figure 2 that at 762 RPM there is no significant upward flow movement which in turn causes more scattering in the data. It is evident that LDV measurements are possible in the latham bowl with the exception of top and bottom of the wall where the surfaces are curved. A curved surface will act as a lens and will distort the laser light.

It was observed that as the angular velocity of the bowl increases the bowl vibrational amplitude increases. In order to quantify the above observation a small piece of electrical wire was taped to the outside wall of the bowl to serve as scattering source for LDV measurements. Since the wire was taped to the bowl, it would vibrate with the same amplitude as the bowl itself. The axial (up and down) velocity of the wire was measured at five different RPM. The mean component of this velocity must be zero since the bowl was firmly held in place. However, the root mean square (RMS) of the velocity fluctuations would represent the strength of vibration. Figure 3 is a plot of the amplitude of vibration as a function of bowl RPM. As expected the vibration becomes stronger with increasing bowl RPM. 
Figure 1. Velocity Vs. Distance from the Outer Wall.

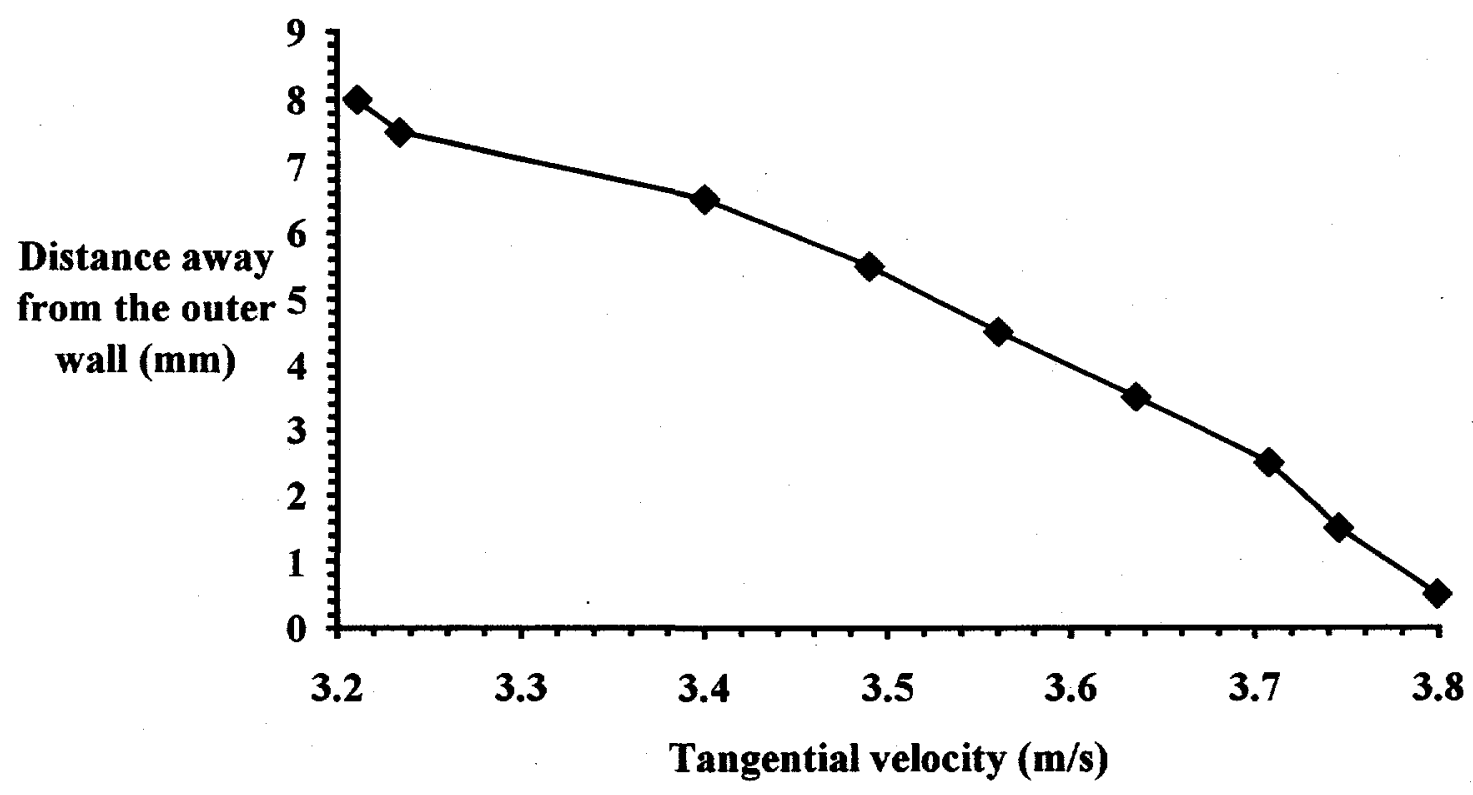

Figure 2. Upward Velocity Vs. Distance from the Wall.

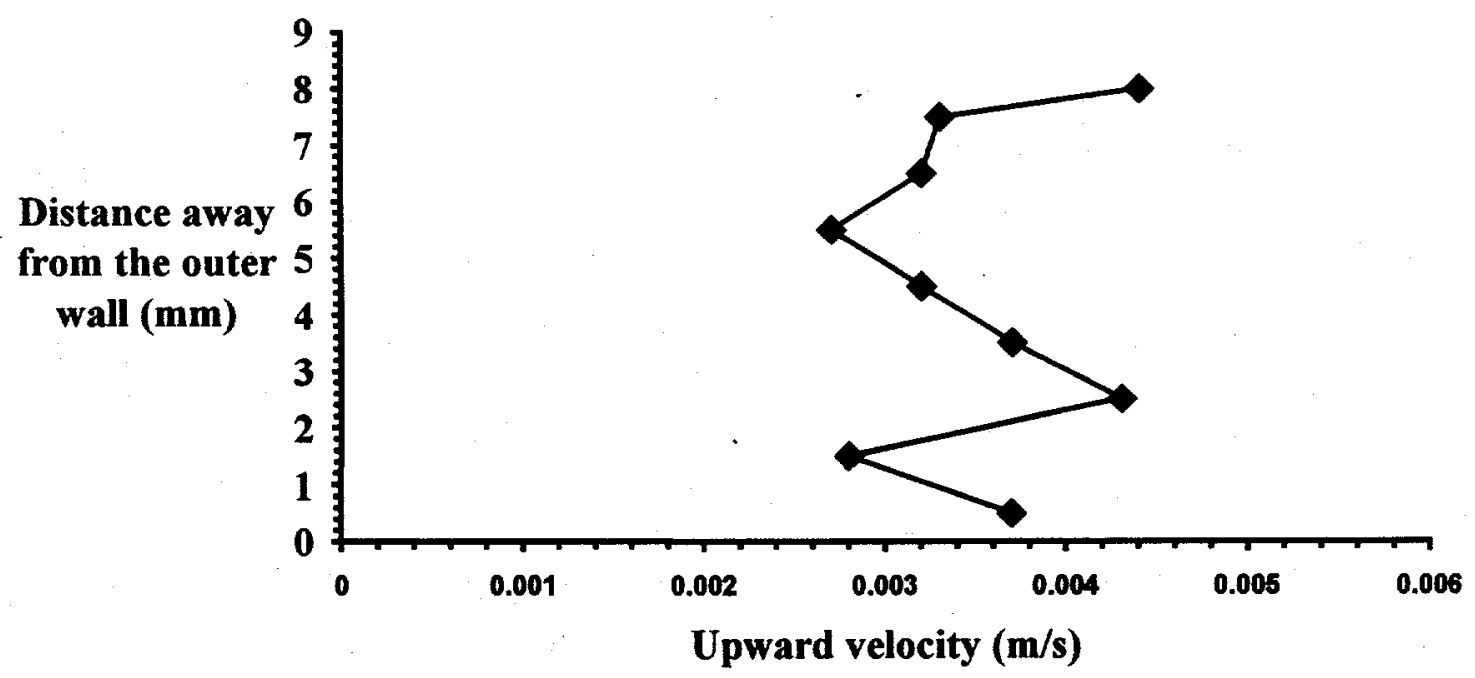


Figure 3. Amplitude of Vibration as a Function of Bowl RPM.

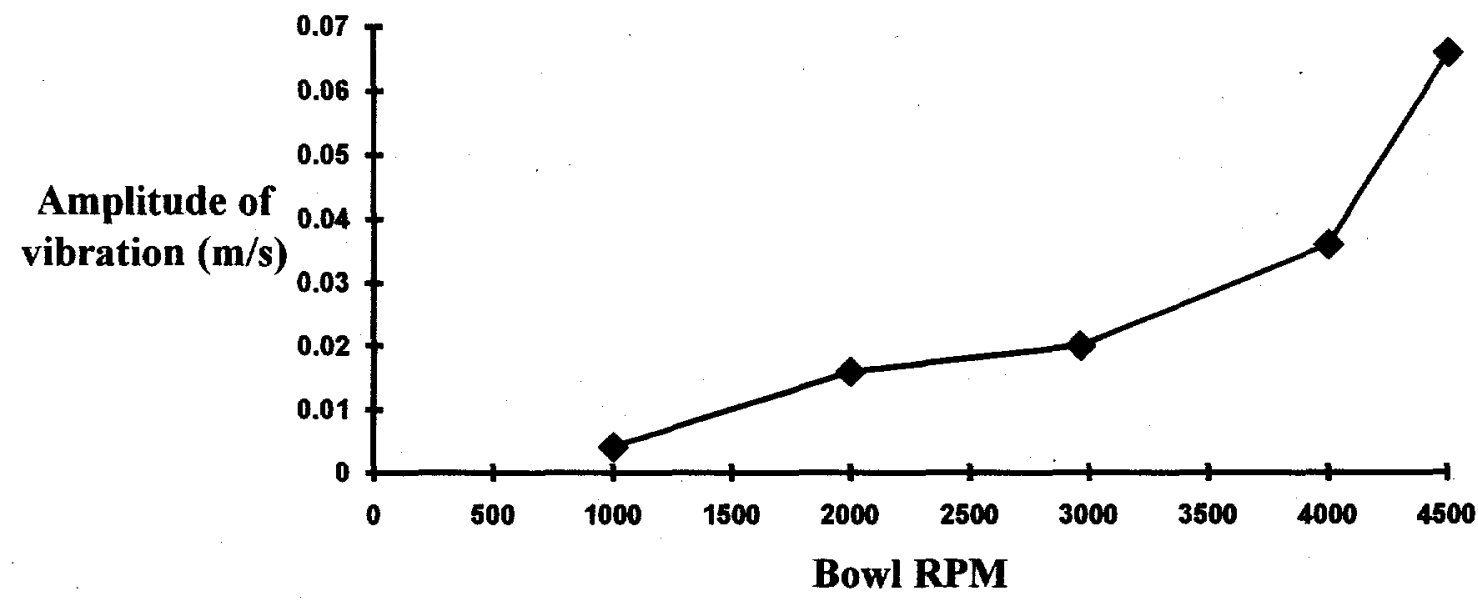

\subsection{SUMMARY}

The feasibility of measuring velocity inside the latham bowl using Laser Doppler Velocimeter is demonstrated. It is possible to take velocity measurements at most areas in the bowl. The difficult locations are areas with high Plexiglas curvature. This difficulty can be partially solved by carefully matching the index of refraction of the bowl material with the working fluid. It is also illustrated that amplitude of vibration of the bowl can be measured as a function of RPM of the bowl. Sources for particles, fluorescent dyes, and optical filters are identified. The fluorescent dyed particles will be used for multiple pulse particle tracking.

\subsection{REFERENCE}

1. William C. Hinds, "Aerosol Technology, ", Wiley Interscience, New York, 1982. 